\title{
EVALUACIÓN AGRONÓMICA Y NUTRICIONAL DEL PASTO RYEGRASS PERENNE TETRAPLOIDE (Lolium perenne) PRODUCIDO EN LECHERÍAS DE LAS ZONAS ALTAS DE COSTA RICA. I. PRODUCCIÓN DE BIOMASA Y FENOLOGÍA
}

\author{
Luis Villalobos ${ }^{1 / *}$,Jorge Ml. Sánchez*
}

\begin{abstract}
Palabras clave: Producción de materia seca, estado fenológico, aprovechamiento, pastos clima templado, Lolium perenne.

Keywords: Dry matter yield, phenological stage, utilization, temperate climate grasses, Lolium perenne.
\end{abstract}

\section{RESUMEN}

Se evaluó la producción de biomasa y la fenología del pasto ryegrass perenne tetraploide (Lolium perenne) a lo largo de un año en muestreos bimensuales, en 4 fincas comerciales de ganado lechero ubicadas en Chicuá de Oreamuno (latitud 0959', longitud 8352', altitud $3090 \mathrm{msnm}$ ), provincia de Cartago. La disponibilidad de materia seca pre y pos-pastoreo, el aprovechamiento por hectárea y su porcentaje de utilización promedio para todas las fincas fueron 4110, 2276 y $1833 \mathrm{~kg} \cdot \mathrm{ha}^{-1}$ por corte y 44,82\%, respectivamente. La edad fenológica y la relación hoja: tallo promedio de las pasturas de ryegrass perenne fueron 2,84 hojas verdes por rebrote, 56:44. Asimismo, la composición promedio de la pradera fue $76,09 \%$ ryegrass, $13,76 \%$ otras gramíneas (principalmente pasto kikuyo), 6,02\% trébol, $1,25 \%$ malezas y $2,88 \%$ material senescente, respectivamente. La mayor producción de biomasa durante marzo y abril puede conservarse para utilizarse durante las épocas críticas de disponibilidad de forraje o cuando por razones climatológicas se reduce el número de horas efectivas de pastoreo. La evaluación de la edad fenológica en que se pastorea el pasto ryegrass perenne, indica que las fincas en la zona de estudio utilizan esta gramínea en un estado en el que

1/ Autor para correspondencia. Correo electrónico: luis.villalobosvillalobos@ucr.ac.cr

\section{ABSTRACT}

Agronomic and nutritional evaluation of perennial tetraploid ryegrass-based pastures (Lolium perenne) in dairy farms in the highlands of Costa Rica. I. Dry matter yield and phenology. Dry matter yield and phenology of perennial tetraploid ryegrass (Lolium perenne)-based-pastures were evaluated in 4 commercial dairy farms in the district of Chicuá, Oreamuno, Cartago (latitude 0959', longitude $83^{\circ} 52^{\prime}, 3090 \mathrm{~m}$ of altitude) every two months during a one-year period. Average pre and post grazing dry matter availability, utilization per hectare and percentage of utilization were 4110 , 2276 and $1833 \mathrm{~kg} \cdot \mathrm{ha}^{-1}$ per grazing cycle and $44.82 \%$, respectively. Average phenological stage and leaf:stem ratio were 2.84 green leaves per regrowth and 56:44. Botanical composition of ryegrass-based-pastures was $76.09 \%$ ryegrass, $13.76 \%$ other grazes (mainly kikuyu grass), $6.02 \%$ clover, $1.25 \%$ weeds and $2.88 \%$ senescent material, respectively. These pastures yield a surplus of DM during March and April, which can be conserved to be fed during the months of less pasture availability. Agronomic data show that the grazing system based on the leaf stage concept is a good tool for managing ryegrassbased-pastures in the highlands of Costa Rica.

Centro de Investigaciones en Nutrición Animal y Escuela de Zootecnia, Universidad de Costa Rica. San José, Costa Rica. 
sus reservas de carbohidratos se han recuperado, lo que permite obtener una producción de biomasa y valor nutricional adecuados para satisfacer las necesidades del ganado lechero de la zona.

\section{INTRODUCCIÓN}

El pasto ryegrass perenne se adapta en zonas entre los 1800 y 3600 msnm, arriba de los 3000 msnm su crecimiento se reduce y los períodos de recuperación se deben prolongar entre 2 y 4 semanas. Los suelos donde crece deben ser de media a alta fertilidad, con un drenaje adecuado y $\mathrm{pH}$ superior a 5,5; es exigente a la nutrición de nitrógeno, fósforo y potasio (Oregon State University 1999). Esta gramínea es poco afectada por plagas y enfermedades; de éstas últimas la más común es la pudrición de la corona causada por Puccinia coronata, sin embargo dichos ataques pueden ser controlados con pesticidas (Vélez et al. 2002).

En términos de producción de materia seca, el fósforo es un nutrimento que se vuelve limitante para un adecuado rebrote debido a que su deficiencia deprime la extracción de nitratos $\left(\mathrm{NO}_{3}\right)$, así como su translocación de las raíces a la parte superior para la producción de aminoácidos (Kim et al. 2003).

De acuerdo con Donaghy y Fulkerson (2001) el manejo adecuado del pastoreo permite producir grandes cantidades de forraje de alta calidad aprovechable para los animales y que pueda persistir por más tiempo. La persistencia de la pastura se optimiza a través de la intensidad de pastoreo reflejado en la altura de los rastrojos pos-pastoreo y la carga animal utilizada, el período de recuperación o rebrote y el de ocupación (Fulkerson y Lowe 2002).

Normalmente se han utilizado diversos criterios para determinar el período de recuperación de la pastura o momento en que ésta debe ser pastoreada tales como días fijos establecidos mediante división de la finca en un número fijo de potreros, índice de área foliar que es difícil de medir y relacionar con las tasas de crecimiento de la planta, altura de la pastura que tiene cierta relación con la MS ofrecida, pero no considera la densidad de la pastura, materia seca ofrecida que tiende a ser complejo de medir directamente por el productor y recientemente el crecimiento y madurez de la hoja (específico de cada especie y tiene una alta relación con el estado vegetativo del pasto) (Fulkerson y Lowe 2002). El objetivo de adecuar los períodos de recuperación de las pasturas con su fenología (número de hojas verdes) es evitar que la planta llegue a un estado de senescencia tal que su valor nutricional disminuya y no se pueda aprovechar una proporción de la materia seca disponible por muerte de hojas (Fulkerson et al. 1993).

El número de hojas que manifiesta la edad fenológica se utiliza en países como Nueva Zelanda y Australia para determinar el momento de cosecha del pasto ryegrass y considera el intervalo mínimo de pastoreo (período requerido para recuperar las reservas de carbohidratos hidrosolubles) el cual en el ryegrass es de más de 2 hojas y el intervalo máximo de pastoreo que se alcanza con el inicio de la senescencia de la hoja más vieja (Fulkerson y Lowe 2002). El ryegrass se considera una planta de 3 hojas ya que la primera hoja en emerger se vuelve senescente conforme la cuarta hoja emerge, dicho ciclo se mantiene después de 3 hojas verdes, por lo que la hoja más vieja morirá de no aprovecharse el forraje (Donaghy y Fulkerson 2001).

La disponibilidad de nutrimentos en el suelo tiene un pequeño efecto sobre el intervalo 
de aparición de las hojas en el ryegrass, por lo que la edad fenológica es un indicador genérico para la utilización de los pastos aplicable a diversas condiciones agronómicas. El contenido de carbohidratos hidrosolubles (CHOS) se considera como el principal factor que influye sobre el crecimiento en pastos y el uso de las reservas que se almacenen en las raíces principalmente, se considera que afecta tanto el período de aparición de las hojas como la persistencia de la pastura, pues en caso de no brindar una recuperación de reservas adecuada a la planta, éstas se agotarán llevando a la pastura a un estado de degradación (Donaghy y Fulkerson 2001).

El número de hojas es un indicador de campo lógico, práctico y conveniente de la recuperación de las reservas de CHOS y de la madurez de la hoja, o de la preparación de la planta para ser pastoreada. El conocimiento del patrón de evolución del número de hojas puede ser usado como una base para el diseño de un sistema de pastoreo controlado. Idealmente se debería utilizar tanto el criterio de la disponibilidad de materia seca como el número de hojas con el fin de ajustar el tiempo de pastoreo (Fulkerson y Lowe 2002).

En condiciones tropicales de altura, debido a que la producción de pasto no presenta una estacionalidad marcada como en países como Nueva Zelanda y Australia, el pasto ryegrass sustenta la producción láctea durante todo el año, sin embargo es importante conocer la producción de biomasa total y su distribución durante el año para planear adecuadamente las necesidades de alimento suplementario que se debe ofrecer a los animales.

Según Donaghy y Fulkerson (2001) la producción de biomasa en pasturas con ryegrass perenne y trébol blanco, que se utilizan en muchos países de clima templado, pueden llegar a 18-20 t.ha ${ }^{-1} \cdot$ año $^{-1}$ de MS bajo condiciones de manejo y ambiente ideales. En condiciones de trópico y sub-trópico se puede obtener producciones de biomasa superiores a 25 t.ha $^{-1} \cdot a_{n} \mathrm{o}^{-1} \mathrm{de}$ MS; sin embargo la persistencia de dichas especies perennes templadas en ocasiones presenta problemas luego de varios años de establecidas (Fulkerson et al. 1993).

Las características ambientales influyen sobre la producción de MS de los pastos y por tanto el establecimiento de especies de clima templado en condiciones tropicales de altura debe asegurar una producción de biomasa relativamente constante y un adecuado aprovechamiento y consumo de los animales. De acuerdo con Donaghy y Fulkerson (2001) la utilización promedio de las pasturas perennes en condiciones de clima templado es de 50-60\% y la pérdida de persistencia es una de las principales limitantes para la producción de ganado de leche.

El llenado físico se considera la principal limitante para obtener un consumo alto de nutrimentos en vacas de leche y dicho mecanismo, de acuerdo con Chilibroste et al. (2000), se puede regular a través de la oferta de un forraje de alto valor nutricional por lo que es recomendable que el pasto utilizado produzca biomasa con una alta concentración de nutrientes y en cantidades adecuadas a las necesidades del hato lechero.

\section{MATERIALES Y MÉTODOS}

Se hicieron 6 muestreos durante un año en 4 fincas comerciales productoras de leche ubicadas en el distrito de San Juan de Chicuá, Oreamuno, Cartago, con latitud 09 $59^{\circ} \mathrm{N}$ y longitud 8352' O y una altitud de $3090 \mathrm{msnm}$. Dicha zona tiene una influencia climática del Caribe muy marcada, se clasifica como bosque pluvial tropical de montano (Holdridge 1947, citado por Janzen 1991), su precipitación promedio es de $1800 \mathrm{~mm}$, el brillo solar promedio es de 5,3 horas por día y las temperaturas mínimas y máximas son $3,6^{\circ} \mathrm{C}$ y $15^{\circ} \mathrm{C}$, respectivamente. Estas últimas presentan pocas variaciones a lo largo del año (Retana 2006).

Las fincas cuentan con sistemas de pastoreo rotacional de tiempo definido esto es, 10 horas de ocupación y 33 a 45 días de recuperación, el pasto predominante es el ryegrass perenne tetraploide (Lolium perenne) con áreas pequeñas de Kikuyo (Kikuyuocloa clandestina). Los animales 
se suplementan con alimento balanceado antes del ordeño con relación a su nivel de producción y etapa de lactancia; además en algunas épocas se suplementa con heno, forrajes de corta como avena forrajera (Avena fatua) fresca o conservada y subproductos de cultivos propios de la zona como papa y zanahoria.

Se realizó un análisis de suelos en cada una de las fincas y se encontraron contenidos minerales propios para un suelo de origen volcánico (andisol). Las bases con valores medios a bajos pero la acidez y el $\mathrm{pH}$ normales, probablemente debido a que son suelos arenosos que se lixivian mucho y que son cultivados de forma intensiva. Asimismo, todas las fincas tenían programas de fertilización establecidos donde los potreros se fertilizan todo el año después del pastoreo en dosis de 3,5 a 6,6 sacos de fertilizante comercial (de $46 \mathrm{~kg}$ ) por cada 1 a 3 potreros por ciclo de pastoreo respectivamente.

En el período de transición de época seca a lluviosa (abril-mayo) se acostumbra aplicar cal en las fincas 1 y 4 , mientras en las fincas 2 y 3 se encala al final de cada 2 ciclos del programa de fertilización. Los fertilizantes más comúnmente utilizados fueron fórmulas completas (10-30-10), Urea, Nitrato de amonio y aplicaciones anuales de enmiendas con cal dolomita. Las dosis de fertilización aplicadas oscilaron entre 200 a 250 kg.ha-1 . año ${ }^{-1}$ de $\mathrm{N}, 30$ a 70 de $\mathrm{P}_{2} \mathrm{O}_{5}$ y 20 a 40 de $\mathrm{K}_{2} \mathrm{O}$.

Se estimó la disponibilidad de MS con la técnica del Botanal® utilizada por Hargraves y Kerr (1978) en 2 potreros durante la mañana y la tarde, por finca en cada una de las visitas realizadas de forma bimensual durante un año. Las mediciones de disponibilidad se realizaron en condiciones pre-pastoreo y pos-pastoreo con el objetivo de estimar el aprovechamiento que hacen los animales del potrero.

La edad fenológica del pasto ryegrass perenne se evaluó por medio del conteo del número de hojas vivas con 50 observaciones por potrero en condiciones pre-pastoreo El número de potreros evaluados durante el año en que se realizó la investigación fue 48; 8 potreros cada 2 meses. Se evaluó la composición estructural de las plantas de ryegrass en una muestra compuesta constituida por múltiples submuestras tomadas al azar en diversos puntos y cortada al ras del suelo para su posterior separación en sus diferentes partes como tallo, hoja y material senescente y secado a $60^{\circ} \mathrm{C}$, para obtener la MS de cada una de las mismas.

En cada uno de los potreros se evaluó la composición botánica de la pastura con 50 observaciones y se asignó las 3 principales especies presentes en orden descendente; dentro de éstas se consideró ryegrass, otras gramíneas principalmente kikuyo (Kikuyuocloa clandestina), Trébol Blanco (Trifolium repens), plantas arvenses (malezas) y material senescente.

La información obtenida para las variables de disponibilidad, edad fenológica, composición estructural de la planta y composición botánica de la pastura se sometió al siguiente modelo con el objeto de buscar efectos significativos $(\mathrm{p} \leq 0,05)$ :

Variables analizadas $=\mu+$ Mes $_{i}+$ Finca $_{j}+$ Mes*Finca $+\mathrm{e}_{\mathrm{ij}}$ $\mu=$ media de las variables estudiadas Mes =i-ésimo efecto de los meses Finca $=\mathrm{j}$-ésimo efecto de las fincas Mes*Finca=interacción meses y fincas $\mathrm{e}=$ residuales del modelo.

Se aplicó la prueba de Duncan para establecer diferencias significativas $(p \leq 0,05)$ entre medias en los diferentes meses y fincas para todas las variables.

\section{RESULTADOS Y DISCUSIÓN}

\section{Producción de biomasa}

La disponibilidad de MS pre y pos-pastoreo, el aprovechamiento por hectárea y su porcentaje de utilización no mostraron diferencias significativas entre las 4 fincas (Cuadro 1). La finca 2 mostró los valores de disponibilidad pre-pastoreo, aprovechamiento por hectárea y porcentaje de aprovechamiento mayores, lo cual puede deberse a que el nivel de suplementación fue el segundo más bajo y a que el tiempo de 
Cuadro 1. Disponibilidad promedio por ciclo de pastoreo de la materia seca (MS) del pasto ryegrass perenne de 4 fincas de ganado lechero evaluadas durante un año, en la zona de San Juan de Chicuá, Oreamuno, Cartago. 2005.

\begin{tabular}{|c|c|c|c|c|}
\hline Finca & $\begin{array}{c}\text { Disponibilidad pre- } \\
\text { pastoreo } \\
\left(\mathrm{kg} \cdot \mathrm{ha}^{-1} \mathrm{MS}\right)\end{array}$ & $\begin{array}{c}\text { Disponibilidad pos- } \\
\text { pastoreo } \\
\left(\mathrm{kg} \cdot \mathrm{ha}^{-1} \mathrm{MS}\right)\end{array}$ & $\begin{array}{c}\text { Aprovechamiento por } \\
\text { hectárea } \\
\left(\mathrm{kg} \cdot \mathrm{ha}^{-1} \mathrm{MS}\right)\end{array}$ & $\%$ Aprovechamiento \\
\hline 1 & $3787(997)^{1}$ & $2088(813)$ & $1699(597)$ & $45,2(12,8)$ \\
\hline 2 & $4510(1236)$ & $2280(763)$ & $2229(1053)$ & $48,4(15,3)$ \\
\hline 3 & 4187 (1572) & 2477 (1342) & $1710(633)$ & $43,2(15,4)$ \\
\hline 4 & 3839 (999) & $2229(838)$ & $1610(767)$ & $41,3(17,1)$ \\
\hline Promedio $^{2}$ & 4110 & 2276 & 1833 & 44,82 \\
\hline
\end{tabular}

1. Desviación estándar entre paréntesis.

2. Los valores corresponden al promedio de 48 muestras.

permanencia en los potreros fue mayor en conjunto con la finca 1, con un total de 21,5 h.día ${ }^{-1}$. Las variables de carga animal, período de ocupación de los potreros, suplementación y tiempo de ordeño son de suma importancia en fincas de ganado de leche para maximizar el aprovechamiento que los animales hacen del pasto disponible y así consumir la mayor cantidad posible durante las horas más frescas del día (Vélez et al. 2002).

La disponibilidad pre-pastoreo promedio del pasto ryegrass perenne fue de $4110 \mathrm{~kg} \cdot \mathrm{ha}^{-1} \mathrm{de}$ MS por ciclo de uso, valor muy cercano a los encontrados por Fulkerson y Donaghy (2001) con 4300 y 2400 kg.ha-1 de MS con fertilización de 200 y 50 $\mathrm{kg} \cdot \mathrm{ha}^{-1} \cdot \mathrm{año}^{-1}$ de $\mathrm{N}$ respectivamente, el efecto de la fertilización se consideró como parte del conjunto de factores que conforman el efecto finca sobre las diversas variables analizadas. Andrade (2006) encontró con el pasto kikuyo un valor promedio de producción de $7238 \mathrm{~kg} \cdot \mathrm{ha}^{-1}$ de MS con aprovechamientos de $20 \%$ debido a la forma rastrera de crecimiento de este pasto tropical.

Las variaciones en disponibilidad pre y pos-pastoreo, aprovechamiento por hectárea y porcentaje de aprovechamiento mostraron una tendencia en relación directa con el comportamiento de la climatología de la zona de estudio (Figura 1). En el período de marzo-abril se

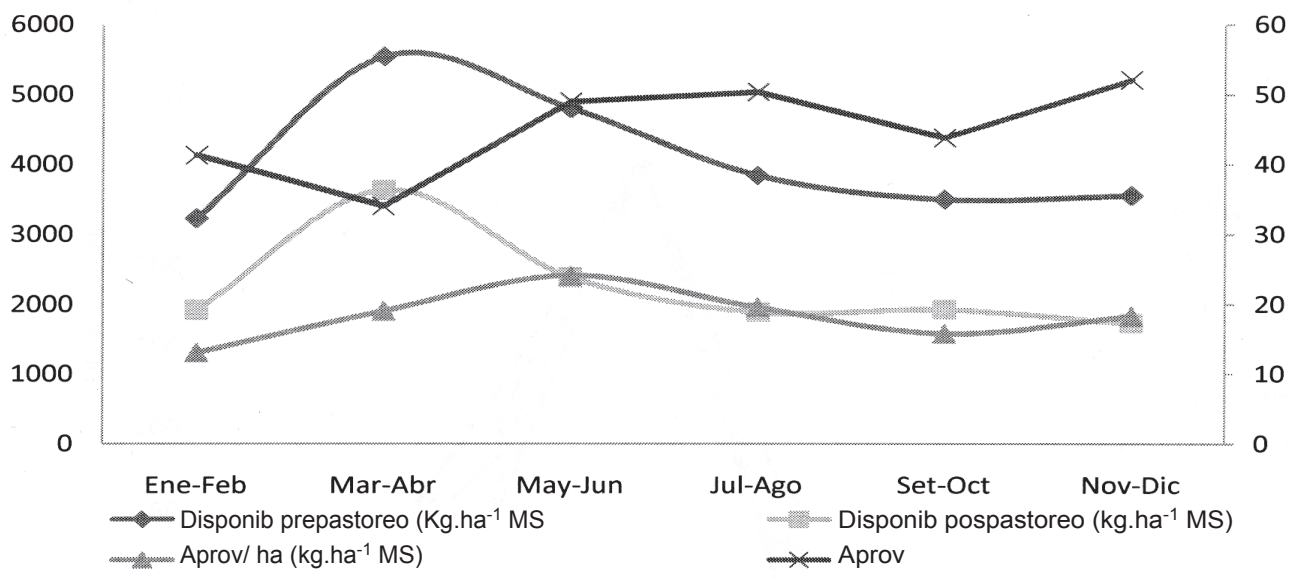

Fig. 1. Variación en la disponibilidad y aprovechamiento del pasto ryegrass perenne a lo largo del año en la zona de San Juan de Chicuá, Oreamuno. 
presentó la mayor disponibilidad de pasto que coincidió con la época de verano en donde hay menos nubosidad en el Pacífico y por lo tanto más brillo solar en la zona ( 6,4 a 8 horas luz por día). A pesar de que la precipitación es baja durante esta época, las características de los suelos volcánicos que retienen más de su peso en agua (Bertsch 1998), les permite producir mayor cantidad de forraje. Asimismo, en dicha época es cuando se registra la mayor velocidad de los vientos $(15,5$ a $\left.18,1 \mathrm{~km} \cdot \mathrm{h}^{-1}\right)$, lo cual genera un gran arrastre de nubes que mantienen el suelo y las plantas con humedad (rocío) para producir sosteniblemente (Retana 2006).

De acuerdo con Oviedo y Ciotti (2004) el ryegrass perenne tiene una tolerancia baja a la anegación, lo anterior se manifiesta en la producción de biomasa baja en setiembre y octubre donde hay una mayor precipitación en la zona y cuyo efecto se extiende hasta el período de noviembre-diciembre debido a la saturación de los suelos que no permite un adecuado movimiento de nutrientes en el suelo. Durante el período enero-febrero se dan las temperaturas mínimas más bajas del año (hasta $3,0^{\circ} \mathrm{C}$ ) y se da una cristalización del rocío sobre las hojas del pasto, fenómeno muy propio de zonas de altura y que a pesar de que el pasto ryegrass lo soporta no deja de ser un factor estresante sobre el desempeño normal de la planta, que se manifiesta en la producción de biomasa durante los 2 primeros meses del año.

La cantidad de pasto residual pos-pastoreo es en parte, producto de la disponibilidad pre-pastoreo; debido a eso, en la época de marzo-abril se presentó un valor mayor $(\mathrm{p} \leq 0,05)$ como resultado de la producción de mayor biomasa. El aprovechamiento por hectárea está influido por la carga animal de cada finca y por cambios climáticos (Cowan y Lowe 1998). En octubre se presenta una precipitación alta que hace a los potreros susceptibles a la compactación por pisoteo y por ser suelos con una densidad aparente baja (Bertsch 1998) se debe controlar la permanencia de los animales en los potreros para que estos no se degraden, ya que los animales presentan dificultades para desplazarse en los terrenos y el forraje se vuelve muy suculento lo que hace que las fincas busquen prácticas de alimentación para suplementar otras fuentes de forraje que permitan mantener un adecuado funcionamiento ruminal (Cruz y Sánchez 2000).

El aprovechamiento por hectárea y el porcentaje de aprovechamiento aumentan en el período de mayo-agosto lo cual puede deberse a las condiciones climáticas favorables y a los valores de producción altos después del bimestre de marzo-abril. En marzo-abril a pesar de contar con una producción de biomasa alta, no se da un adecuado aprovechamiento de dicha biomasa ya que hay una diferencia de 10 unidades porcentuales con respecto al valor promedio de aprovechamiento, esto representa alrededor de $600 \mathrm{~kg}$ de MS que los animales dejan de consumir por cada hectárea cosechada en esa época. Debido a lo anterior es que se debe implementar prácticas de manejo de las pasturas que permitan un mayor aprovechamiento de la biomasa disponible en las épocas de abundancia para su utilización en épocas críticas debido al déficit de forraje o a la dificultad de pastoreo de los animales por la condición del suelo.

De acuerdo con Donaghy y Fulkerson (2001) el promedio de utilización del pasto ryegrass perenne se estima en 50 a $60 \%$ en Nueva Zelanda, valores que son superiores a los encontrados en esta investigación, sin embargo dichos sistemas se basan únicamente en forraje y no se brinda suplementos como es habitual en nuestro país y que genera un efecto sustitutivo del consumo del pasto. Se encontró un efecto significativo del mes para la disponibilidad pre y pos pastoreo ( $\mathrm{p}=0,0011$ y 0,0002 respectivamente), sin embargo a pesar de las variaciones mencionadas entre cada uno de los meses para la disponibilidad, no hubo efecto significativo $(\mathrm{p} \leq 0,05)$ del mes sobre el aprovechamiento por hectárea y el porcentaje de aprovechamiento. 


\section{Edad fenológica}

El número de hojas verdes del pasto ryegrass perenne fue influenciado en forma altamente significativa por el efecto del mes $(\mathrm{p} \leq 0,0001)$ (Cuadro 2). Retana (2006) indica que en la zona de Chicuá existen 2 épocas bien definidas que comprenden de mayo a octubre y de noviembre a abril, en diciembre comienza la temporada de los vientos del norte y frentes fríos que barren

Cuadro 2. Edad fenológica del pasto ryegrass perenne durante un año en la zona de San Juan de Chicuá, Oreamuno, Cartago. 2005.

\begin{tabular}{lc}
\hline Meses & $\begin{array}{c}\text { Edad fenológica } \\
\text { (número de hojas) }\end{array}$ \\
\hline Enero-Febrero & $3,0412^{\mathrm{a}}(0,18)^{1}$ \\
Marzo-Abril & $2,9400^{\mathrm{b}}(0,09)$ \\
Mayo-Junio & $2,7225^{\mathrm{c}}(0,14)$ \\
Julio-Agosto & $2,6887^{\mathrm{c}}(0,20)$ \\
Setiembre-Octubre & $2,7529^{\mathrm{c}}(0,10)$ \\
Noviembre-Diciembre & $2,8650^{\mathrm{b}}(0,07)$ \\
Promedio $^{2}$ & 2,84 \\
\hline
\end{tabular}

$\mathrm{a}, \mathrm{b}, \mathrm{c}$ medias con letras diferentes dentro de una misma columna difieren entre sí, según la prueba de Duncan $(\mathrm{p} \leq 0,05)$

1. Desviación estándar entre paréntesis.

2. El valor corresponde al promedio de 48 muestras (potreros), cada una compuesta de 50 observaciones. parcialmente la nubosidad, lo que hace que aumente el brillo solar y las plantas produzcan una cantidad mayor de hojas. En marzo y abril disminuyó la edad fenológica promedio y de mayo a octubre se presentaron los valores más bajos $(\mathrm{p} \leq 0,05)$. Las variaciones encontradas en la edad fenológica de las plantas permiten a los productores mejorar sus prácticas de manejo de pasturas con el objetivo de aprovechar mejor el recurso forrajero para lo cual se puede diferir la rotación de algunos potreros para utilizarlos en sistemas de conservación, como el ensilaje o el henilaje, o bien acortar los períodos de recuperación de las pasturas.

Hubo un efecto significativo $(\mathrm{p}=0,0237) \mathrm{de}$ la finca sobre la edad fenológica y de la planta la interacción mes*finca fue altamente significativa $(p \leq 0,0001)$. El manejo de cada una de las fincas durante el período de recuperación afectó $(\mathrm{p} \leq 0,05)$ en el número de hojas (Cuadro 3). Las fincas 1, 2 y 3 mostraron valores similares y la finca 4 tuvo el número de hojas menor, lo cual puede deberse a que es la finca con el período de recuperación más corto (32 días) y por tanto los animales consumen un forraje más suculento que el de las otras 3 .

De acuerdo con Retana (2006) la ubicación de las fincas 1 y 2 permite que reciban una mayor influencia del clima de la región Caribe mientras las fincas 3 y 4 del Pacífico, lo cual puede producir diferencias en el crecimiento de las plantas y los períodos de recuperación. Todas las fincas se encontraron dentro del rango de edad fenológica

Cuadro 3. Edad fenológica promedio del pasto ryegrass perenne en 4 fincas de ganado de leche en la zona de San Juan de Chicuá, Oreamuno, Cartago. 2005.

\begin{tabular}{ccc}
\hline Finca & Edad fenológica ${ }^{2}$ (número de hojas) & Días de recuperación \\
\hline 1 & $2,8692^{\mathrm{a}}(0,22)^{1}$ & 45 \\
2 & $2,8858^{\mathrm{a}}(0,12)$ & 35 \\
3 & $2,8108^{\mathrm{ab}}(0,14)$ & 35 \\
4 & $2,7764^{\mathrm{b}}(0,24)$ & 32 \\
Promedio $^{2}$ & 2,84 & 36,75 \\
\hline
\end{tabular}

a, b, c medias con letras diferentes dentro de una misma columna difieren entre sí, según la prueba de Duncan ( $\mathrm{p} \leq 0,05)$.

1. Desviación estándar entre paréntesis.

2. El valor corresponde al promedio de 48 muestras (potreros), cada una compuesta de 50 observaciones. 
sugerido por Fulkerson y Lowe (2002) (Cuadro 3), el cual es mínimo de 2 hojas. En este estado que las reservas de carbohidratos hidrosolubles se han recuperado para que la planta sea pastoreada sin limitar el rebrote posterior. El número máximo sugerido por los autores citados es 3 hojas, después de este estado emerge la cuarta hoja y la primera hoja en emerger se vuelve senescente y la calidad de la pastura declina. En la Figura 2 se presenta la frecuencia de observaciones para el conteo del número de hojas en que se pastorea el ryegrass perenne en las 4 fincas estudiadas en la zona de Chicuá. Se observa que la frecuencia mayor se encontró en 3 hojas verdes por rebrote, por lo que las 4 fincas utilizan el ryegrass perenne a una edad fenológica adecuada que le permite a la planta acumular reservas con las ventajas que esto genera sobre la persistencia, la productividad, utilización y calidad nutricional de las pasturas (Donaghy y Fulkerson 2001).

\section{Composición estructural de las plantas de ryegrass perenne}

La composición estructural del pasto ryegrass perenne mostró un promedio de $56,08 \%$ de hoja a lo largo del año y dicho componente fue afectado de forma significativa por el mes $(\mathrm{p}=0,0300)$ (Cuadro 4). El porcentaje de hoja del pasto ryegrass perenne fue superior al encontrado por Andrade (2006) para el pasto kikuyo con $32 \%$, asimismo el porcentaje de tallos y material senescente del ryegrass perenne fue $43,92 \%$, valor inferior al del pasto kikuyo que es $68 \%$.

Cuadro 4. Composición estructural de la planta de ryegrass perenne durante un año, en potreros de la zona de San Juan de Chicuá, Oreamuno, Cartago. 2005.

\begin{tabular}{ll}
\hline Meses & $\begin{array}{c}\text { Relación hoja-tallo } \\
\text { (\%hoja) }\end{array}$ \\
\hline Enero-Febrero & $52,55: 47,45^{\mathrm{b}}(4,60)^{1}$ \\
Marzo-Abril & $57,78: 42,22^{\mathrm{ab}}(5,69)$ \\
Mayo-Junio & $60,79: 39,21^{\mathrm{a}}(7,65)$ \\
Julio-Agosto & $55,62: 44,38^{\mathrm{ab}}(5,06)$ \\
Setiembre-Octubre & $52,03: 47,97^{\mathrm{b}}(4,61)$ \\
Noviembre-Diciembre & $57,73: 42,27^{\mathrm{ab}}(2,38)$ \\
Promedio $^{2}$ & $56,08: 43,92$ \\
\hline
\end{tabular}

a, b, c medias con letras diferentes dentro de una misma columna difieren entre sí, según la prueba de Duncan $(\mathrm{p} \leq 0,05)$.

1. Desviación estándar entre paréntesis.

2. El valor corresponde al promedio de 48 muestras (8 por muestreo).

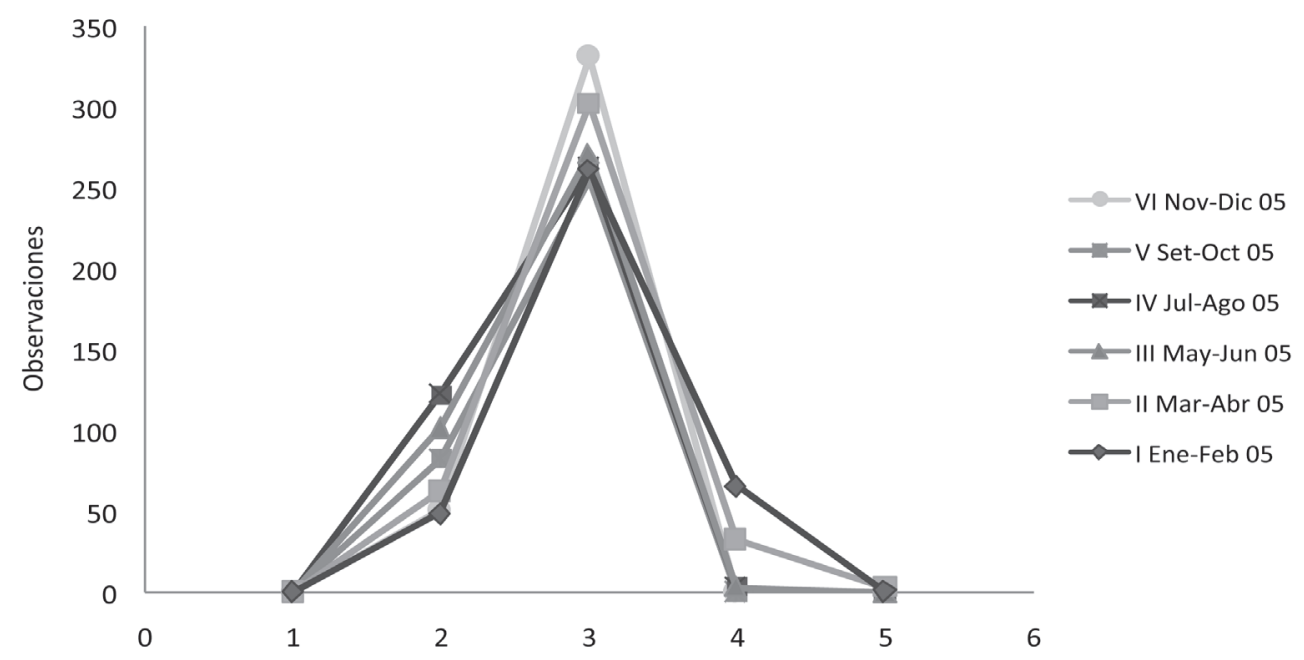

Fig.2. Distribución de la frecuencia de observaciones del número de hojas verdes por rebrote en el pasto ryegrass perenne en 6 muestreos realizados durante un año. 
En enero y febrero el porcentaje de hojas en las pasturas disminuyó, durante esta época la producción de biomasa fue menor por lo que la menor proporción de hojas puede deberse al estrés causado por la radiación solar alta, la precipitación menor, las temperaturas mínimas más bajas en el año y la cristalización del rocío en las hojas del pasto ryegrass. Con el inicio de las lluvias la proporción de hojas aumentó, lo que puede asociarse a una extracción mayor de los nutrientes que tienen una mayor movilidad en el suelo (Bertsch 1998).

De julio a octubre hubo una disminución significativa $(\mathrm{p} \leq 0,05)$ en la relación hoja-tallo de las pasturas, en dicha época la precipitación y nubosidad mayor provocan saturación hídrica en los suelos, por lo que el rebrote de la planta es más lento. Al final de año la radiación solar aumenta en la zona, lo que estimula una mayor producción de hojas. La relación entre hojas, tallos y material senescente es un indicador de qué tan asertiva es la escogencia del ciclo de uso de las pasturas, basándose en las necesidades de los animales ya que la mayor parte de los nutrientes se concentran en las hojas (Kim et al. 2003).

Las fincas no afectaron significativamente la composición estructural de la planta, ya que según Vélez et al. (2002), dicha característica es un indicador de las adaptaciones morfológicas de la especie de pasto. La altura del pasto ryegrass perenne al momento del pastoreo no fue uniforme, probablemente por la competencia inter e intraespecífica normal en una pastura, sin embargo debe considerarse que los productores han adaptado el ryegrass a las condiciones de manejo de las fincas, lo cual se evidencia en que la edad fenológica se encuentra dentro de los rangos recomendados.

\section{Composición botánica de las pasturas de ryegrass perenne}

El porcentaje de pasto ryegrass perenne no mostró cambios significativos $(\mathrm{p} \geq 0,05)$ a lo largo del año, ni fue afectado por la finca, el mes de muestreo o la interacción de ambos (Cuadro 5). A pesar de esto su presencia disminuye en la época seca, lo cual en otras latitudes es el fundamento de su utilización debido a ser una especie activa durante el invierno y con crecimiento pobre en veranos calientes y secos donde su producción disminuye de forma estacional (Kemp et al. 2002). La presencia de otras gramíneas mostró un efecto significativo por la interacción mes*finca $(\mathrm{p}=0,0280)$, dicho grupo de gramíneas

Cuadro 5. Composición botánica de las pasturas de ryegrass perenne en la zona de San Juan de Chicuá, Oreamuno, Cartago durante el 2005.

Porcentaje presente en la pastura (\%)

\begin{tabular}{|c|c|c|c|c|c|}
\hline Meses & Ryegrass & Otras gramíneas & Trébol & Malezas & $\begin{array}{c}\text { Material } \\
\text { senescente }\end{array}$ \\
\hline Ene-Feb & 67,72 & 9,29 & $16,17^{\mathrm{a}}$ & 1,01 & $5,81^{\mathrm{a}}$ \\
\hline Mar-Abr & 80,30 & 9,81 & $3,39^{b}$ & 0,49 & $6,01^{\mathrm{a}}$ \\
\hline May-Jun & 77,21 & 16,99 & $1,69^{b}$ & 1,38 & $2,73^{b}$ \\
\hline Jul-Ago & 74,80 & 18,61 & $4,49^{b}$ & 1,24 & $0,86^{\mathrm{bc}}$ \\
\hline Set-Oct & 75,87 & 12,22 & $8,30^{\mathrm{b}}$ & 2,02 & $1,59^{b c}$ \\
\hline Nov-Dic & 80,63 & 15,65 & $2,05^{\mathrm{b}}$ & 1,40 & $0,27^{\mathrm{c}}$ \\
\hline Promedio $^{1}$ & 76,09 & 13,76 & 6,02 & 1,25 & 2,88 \\
\hline
\end{tabular}

a, b, c medias con letras diferentes dentro de una misma columna difieren entre sí, según la prueba de Duncan ( $\mathrm{p} \leq 0,05)$.

1. Los valores corresponden al promedio de 48 muestras, cada una compuesta de 50 observaciones. 
se compuso principalmente de pasto kikuyo, el cual es más susceptible al brillo solar y la cristalización del rocío durante la época de verano, que el pasto ryegrass.

La presencia de trébol blanco en las pasturas de ryegrass perenne es afectada de forma significativa y altamente significativa por el mes $(\mathrm{p}=0,0006)$ y la interacción mes*finca $(\mathrm{p} \leq 0,0001)$, respectivamente. El trébol detiene su crecimiento a temperaturas inferiores a $8^{\circ} \mathrm{C}$ por lo que es de utilidad para brindar forraje de alta calidad durante la época seca compensar el aumento en material senescente de los 4 primeros meses del año (Kemp et al. 2002) (Cuadro 5).

Las prácticas de manejo de cada finca afectan de forma significativa $(\mathrm{p}=0,0002)$ la presencia de malezas, pues a pesar de no variar a lo largo del año, cada finca cuenta con diferentes métodos de control en potreros. Las principales especies encontradas en los potreros fueron: Hydrocotile sp., Poa апnиa, Taraxacum sp. y Rumex sp.

El porcentaje de material senescente en las pasturas se vio afectado de forma altamente significativa por el mes $(\mathrm{p} \leq 0,0001)$ y de forma significativa por la finca $(\mathrm{p}=0,0167)$ y la interacción mes*finca $(\mathrm{p}=0,0120)$. Se puede considerar un indicador de efectividad del aprovechamiento que se hace de las pasturas y si es necesario hacer ajustes en los días de recuperación del pasto, ya que su presencia aumenta de forma significativa durante los meses de mayor producción de biomasa.
La finca 2 mostró el porcentaje mayor de ryegrass en las pasturas y la finca 4 el porcentaje menor, ésta a su vez mostró una presencia mayor de otras gramíneas, trébol y malezas, lo cual puede deberse a que el período de recuperación menor vuelve más vulnerable a la invasión de otras especies que se deben controlar por medios químicos y culturales (Cuadro 6).

\section{CONCLUSIONES Y RECOMENDACIONES}

La producción de biomasa del pasto ryegrass perenne aumenta significativamente $(\mathrm{p} \leq 0,05)$ en la época de mayor radiación solar y se mantiene relativamente constante el resto del año, por lo que las fincas deben utilizar el excedente para las épocas críticas debido al exceso de humedad en los suelos que limita el pastoreo adecuado en los terrenos. La disponibilidad pre-pastoreo promedio fue de $4100 \mathrm{~kg} \cdot \mathrm{ha}^{-1}$ por corte de MS, la cual tiene un aprovechamiento de $45 \%$.

A pesar de contar con prácticas de manejo de pasturas diferentes, las 4 fincas mostraron valores similares en las variables de disponibilidad de materia seca, el porcentaje de aprovechamiento promedio fue de $44,82 \%$, el cual disminuyó en los meses de mayor producción debido a que las cargas animales se mantienen relativamente constantes durante todo el año por lo que se sugiere diferir terrenos en dicha época con el fin de conservar forraje para elaborar

Cuadro 6. Composición botánica de las pasturas de ryegrass perenne en 4 fincas de ganado de leche en la zona de San Juan de Chicuá, Oreamuno, Cartago. 2005.

\begin{tabular}{|c|c|c|c|c|c|}
\hline \multirow[b]{2}{*}{ Finca } & \multicolumn{5}{|c|}{ Porcentaje presente en la pastura $^{1}(\%)$} \\
\hline & Ryegrass & Otras gramíneas & Trébol & Malezas & Senescente \\
\hline 1 & 78,27 & $14,75^{a b}$ & $4,31^{\mathrm{b}}$ & $0,32^{b}$ & $2,35^{a b}$ \\
\hline 2 & 84,40 & $6,73^{b}$ & $4,26^{\mathrm{b}}$ & $0,85^{\mathrm{b}}$ & $3,76^{\mathrm{a}}$ \\
\hline 3 & 77,55 & 13,33 ab & $4,56^{\mathrm{b}}$ & $0,59^{\mathrm{b}}$ & $3,97^{\mathrm{a}}$ \\
\hline 4 & 63,07 & $20,95^{\mathrm{a}}$ & $11,40^{\mathrm{a}}$ & $3,28^{a}$ & $1,30^{\mathrm{b}}$ \\
\hline
\end{tabular}

1. Los valores corresponden al promedio de 12 muestras por finca (potreros), cada una compuesta de 50 observaciones. $\mathrm{a}, \mathrm{b}, \mathrm{c}$ medias con letras diferentes dentro de una misma columna difieren entre sí, según la prueba de Duncan ( $\mathrm{p} \leq 0,05)$. 
henilaje o ensilaje y aprovechar las condiciones favorables del clima de la época menos lluviosa.

La edad fenológica del pasto ryegrass perenne aumentó en los meses de producción de mayor biomasa por lo que se debe adecuar el manejo de las pasturas a las condiciones variables del clima durante el año. Las 4 fincas tienen valores adecuados de edad fenológica con base en lo que se recomienda en la literatura universal a pesar de las diferencias en tamaño, densidad y altura de las plantas dentro de los potreros evaluados. Es importante que el pasto ryegrass perenne se utilice entre 2 y 3 hojas para asegurar una disponibilidad, persistencia y calidad nutritiva.

El pasto ryegrass perenne tiene un $56,08 \%$ de hoja promedio en su composición estructural, lo cual es superior a pastos tropicales de altura tal como el kikuyo. Su variación durante el año se debe a factores climáticos estresantes para el adecuado rebrote de la planta. Las 4 fincas analizadas tuvieron valores similares lo que sugiere que en todas se maneja el pasto en forma apropiada.

El porcentaje de ryegrass perenne no varió significativamente $(\mathrm{p} \geq 0,05)$ en el año pero disminuyó en época seca, así como las otras gramíneas, compuestas principalmente de pasto kikuyo. La presencia de malezas en los potreros tiene una relación directa con las prácticas de manejo de cada finca y el material senescente aumentó en los meses de mayor producción de biomasa debido al menor aprovechamiento que se hace del recurso forrajero excedente. Debido a lo anterior las tecnologías de conservación de forrajes se deben implementar en las fincas de la zona como prácticas rutinarias.

\section{AGRADECIMIENTOS}

Los autores expresan su agradecimiento a los propietarios de las fincas donde se realizó esta investigación: Sr. Rolando Ferreto, Sra. Gabriela Arrea, Sr. Noré Gómez y Sr. Bernardo Gómez (q.d.D.g.), así como a los trabajadores de las fincas por la colaboración brindada durante el año en que se realizó la investigación.

\section{LITERATURA CITADA}

ANDRADE M. 2006. Evaluación de técnicas de manejo para mejorar la utilización del pasto kikuyo (Pennisetum clandestinum_Hochst. Ex Chiov) en la producción de ganado lechero en Costa Rica. Tesis de licenciatura, Universidad de Costa Rica, San José, Costa Rica. $225 \mathrm{p}$.

BERTSCH F. 1998. La fertilidad de los suelos y su manejo. San José, Costa Rica. Asociación Costarricense de la Ciencia del Suelo. 157 p.

CHILIBROSTE P., TAMMINGA S., BOER. 2000. Duration of regrowth of Ryegrass (Lolium perenne) Effects on grazing behavior, intake, rumen fill, and fermentation of lactating dairy cows. Journal of Dairy Science 83:984-995

COWAN R.T., LOWE. 1998. Tropical and subtropical grass management and quality. In: Grass for dairy cattle. (C)CAB International:101-135.

CRUZ M., SÁNCHEZ J. 2000. La fibra en la alimentación del ganado lechero. Nutrición Animal Tropical 6:3974.

DONAGHY D., FULKERSON B. 2001. Principles for developing an effective grazing management system for ryegrass-based pastures. Tasmanian Institute of Agricultural Research, Burnie, Tasmania. 10 p.

FULKERSON W.J., DONAGHY D.J. 2001. Plant-soluble carbohydrate reserves and senescence key criteria for developing an effective grazing management system for ryegrass-based pastures: a review. Australian Journal of Experimental Agriculture. 41:261-275.

FULKERSON W.J., LOWE K.F. 2002. Grazing Management. Forages and Pastures. 1142-1149.

FULKERSON W.J., LOWE K.J., AYRES J.F., LAUNDERS T. 1993. Winter pastures and crops. Tropical Grasslands. 27:162-179.

HARGRAVES J.N.G., KERR J.D. 1978. Botanal: a comprehensive sampling and computing procedure for estimating pasture yield and composition. II. Computational package. Division of Tropical Crops and Pastures, Tropical Agronomy, CSIRO, Australia. Technical Memorandum N ${ }^{\circ} .9$.

JANZEN D.H. 1991. Historia natural de Costa Rica. $1^{\text {a }}$ ed. Editorial de la UCR. San José, Costa Rica. 822 p.

KEMP P.D., MATTHEW C., LUCAS R.J. 2002. Pasture species and cultivars. In: New Zealand: Pasture and 
Crop Science. Oxford University. South Melbourne, Australia. 323 p.

KIM T.W., JUNG W.J., LEE B., YONEYAMA T., KIM H., KIM K. 2003. P effects on $\mathrm{N}$ uptake and remobilization during regrowth of Italian ryegrass (Loluim multiflorum). Environmental and Experimental Botany. 50:233-242.

OREGON STATE UNIVERSITY. 1999. Brochure: Perennial Ryegrass. Oregon Ryegrass Growers Seed Commission. Consultado el 11/05/2005 In: www. ryegrass.com.
OVIEDO M.B., CIOTTI E.M. 2004. Efecto del anegamiento temporario en el establecimiento de Lolium multiflorum Lam. Consultado el 11/05/2005 In: http://agr.unne.edu.ar/Extensión/Res2004/Forrajes/ Forr-001.pdf.

RETANA J. 2006. Climatología de la región del distrito de San Juan de Chicuá y el Volcán Irazú. Informe anual del Instituto Meteorológico Nacional. 3 p.

VÉLEZ M., HINCAPIE J.J., MATAMOROS I., SANTILLAN R. 2002. Producción de Ganado Lechero en el Trópico. Cuarta edición. Zamorano Academic Press, Zamorano, Honduras. 326 p. 